\title{
Asynchronous Sequential Symbol Synchronizers based on Pulse Comparison by Positive Transitions at Half Bit Rate
}

\author{
Antonio D. Reis ${ }^{1,2}$, José F. Rocha ${ }^{1}$, Atilio S. Gameiro ${ }^{1}$, José P. Carvalho ${ }^{2}$ \\ ${ }^{1}$ Dep. de Electrónica e Telecomunicações / Instituto de Telecomunicações, Universidade de Aveiro, 3810 Aveiro, Portugal \\ ${ }^{2}$ Dep. de Fisica / U. D. Remota, Universidade da Beira Interior Covilhã, 6200 Covilhã, Portugal
}

\begin{abstract}
This work presents the asynchronous sequential symbol synchronizers based on pulse comparison by positive transitions at half bit rate. Their performance will be compared with the reference asynchronous symbol synchronizers based on pulse comparison by both transitions at bit rate.

For the reference and proposed variants, we consider two versions which are the manual $(\mathrm{m})$ and the automatic (a).

The objective is to study the four synchronizers and evaluate their output jitter UIRMS (Unit Interval Root Mean Square) versus input SNR (Signal Noise Ratio).

Keywords - Synchronism, Digital Communications
\end{abstract}

\section{INTRODUCTION}

This work studies the asynchronous sequential symbol synchronizer based on pulse comparison operating by positive transitions at half bit rate (ap/2). Their jitter is compared with the reference asynchronous synchronizers operating by both transitions at bit rate $(\mathrm{ab})[1,2]$.

For both, reference and proposed variant, we consider the versions manual (m) and automatic (a) $[3,4,5,6,7]$.

The difference between the reference and proposed synchronizer is in the symbol phase comparator since the others blocks are similar. The phase comparator compares the input variable pulse duration $\mathrm{Pv}$ with the intern reference fixed pulse duration Pf and the error pulse Pe synchronizes the VCO (Voltage Controlled Oscillator) $[8,9]$.

The synchronizer regenerates the data, recovering a clock (VCO) that samples and retimes the data $[10,11,12,13]$.

Fig. 1 shows the blocks of the general symbol synchronizer.

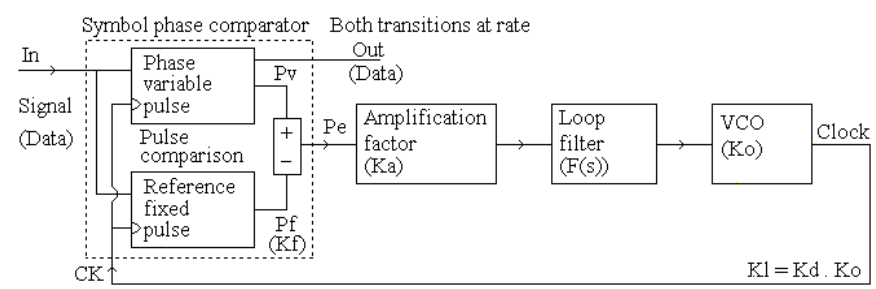

Fig.1 Synchronizer based on pulse comparison

$\mathrm{Kf}$ is the phase comparator gain, $\mathrm{F}(\mathrm{s})$ is the loop filter, Ko is the VCO gain and $\mathrm{Ka}$ is the loop amplification factor that controls the root locus and then the loop characteristics.

In priori and actual-art state was developed various synchronizers, now is necessary to know their performance.

The motivation of this work is to create new synchronizers and to evaluate their performance with noise. This contribution increases the knowledge about synchronizers.
Following, we present the reference variant, asynchronous sequential symbol synchronizers based on pulse comparison by both transitions at bit rate, with versions manual (ab-m) and automatic (ab-a). Next, we present the proposed variant, asynchronous sequential symbol synchronizer based on pulse comparison by positive transitions at half bit rate, with versions manual (ap-m/2) and automatic (ap-a/2).

After, we present the design and tests. Then, we present the results. Finally, we present the conclusions.

\section{REFERENCE BY BOTH AT RATE}

The normal reference, asynchronous sequential symbol synchronizers based on pulse comparison operating by both transitions at bit rate has two versions which are the manual (ab-m) and the automatic (ab-a) [1, 2].

The versions difference is in the phase comparator, the variable pulse $\mathrm{Pv}$ is common but the fixed $\mathrm{Pf}$ is different.

\section{A. Reference by both at rate manual (ab-m)}

The block Pv, shown below, produces a variable pulse Pv between the input bits and VCO. The manual adjustment delay with Exor produces a manual fixed pulse Pf (Fig.2).

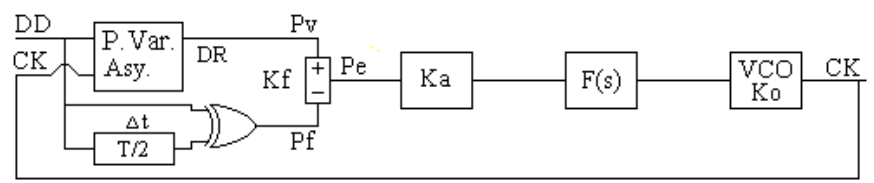

Fig.2 Asynchronous by both at rate and manual (ab-m)

The comparison between the pulses $\mathrm{Pv}$ and $\mathrm{Pf}$ provides the error pulse Pe that forces the VCO to synchronize the input. The block $\mathrm{Pv}$ is an asynchronous circuit (Fig.3).

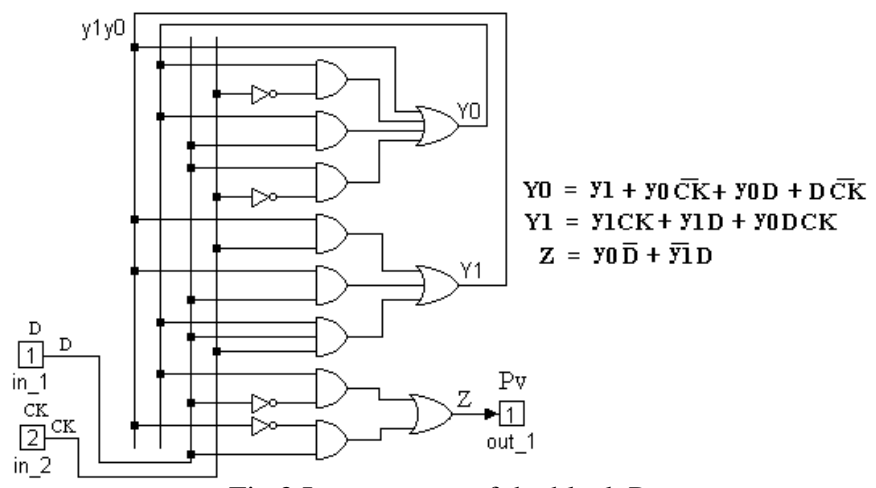

Fig. 3 Intern aspect of the block Pv

\footnotetext{
${ }^{1 / 2} \mathrm{UA}-\mathrm{UBI}$
} 
Fig.4 shows the waveforms of the reference manual (equal to the corresponding synchronous version) [3].

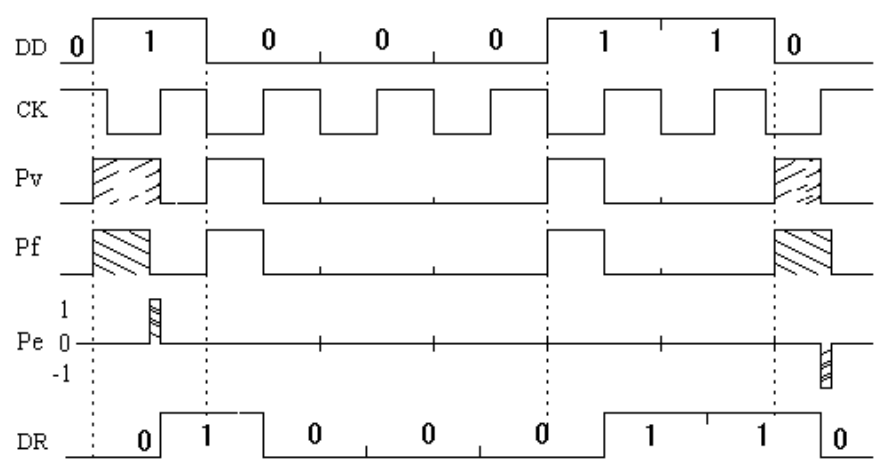

Fig.4 Waveforms of the asynchronous by both at rate manual

The error pulse Pe diminishes during the synchronization time and disappear at the equilibrium point.

\section{B. Reference by both at rate automatic (ab-a)}

The block Pv, common with anterior, produces the variable pulse $\mathrm{Pv}$ between input and VCO. The block Pf, shown below, produces the comparison fixed pulse Pf (Fig.5).

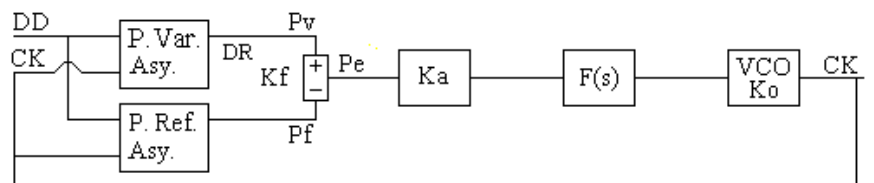

Fig.5 Asynchronous by both at rate and automatic (ab-a)

The comparison between the pulses $\mathrm{Pv}$ and $\mathrm{Pf}$ provides the error pulse Pe that forces the VCO to follow the input. The block Pf is an asynchronous circuit (Fig.6).

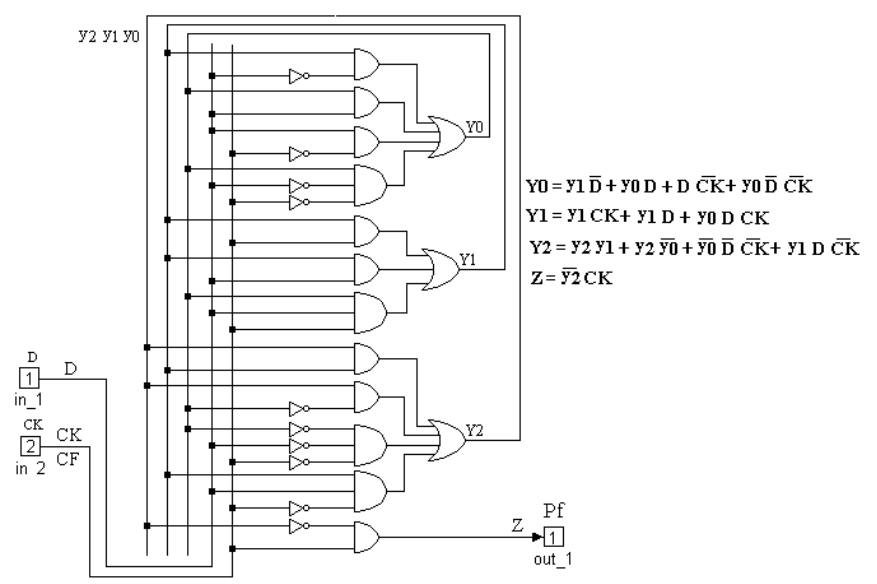

Fig.6 Intern aspect of the block Pf

Fig.7 shows the waveforms of the reference automatic (equal to the corresponding synchronous version) [3].

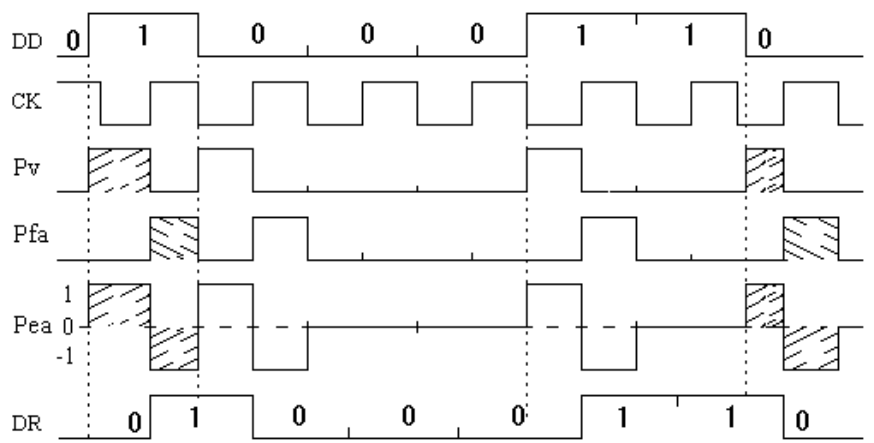

Fig.7 Waveforms of the asynchronous by both at rate automatic

The error pulse Pe don't disappear, but the variable area Pv is equal to the fixed Pf at the equilibrium point.

\section{PROPOSED BY POSITIVE AT HALF RATE}

The new proposed, asynchronous sequential symbol synchronizers based on pulse comparison operating by positive transitions at half bit rate has also two versions namely the manual (ap-m/2) and the automatic (ap-a/2) [3, 4].

The versions difference is in the phase comparator, the variable pulse $\mathrm{Pv}$ is common but the fixed $\mathrm{Pf}$ is different.

\section{A. Proposed by positive half rate manual (ap-m/2)}

The block Pv produces the variable pulse $\mathrm{Pv}$ between input transitions and VCO. The manual adjustment delay T/2 with AND produces a fixed pulse Pf (Fig.8).

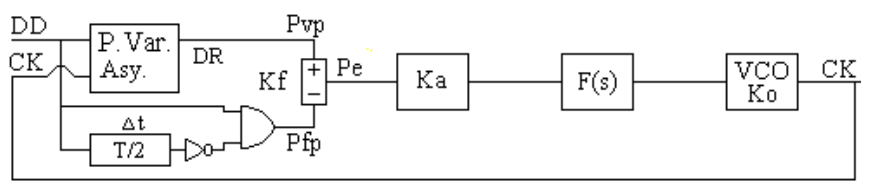

Fig.8 Asynchronous by positive at half rate and manual (ap-m/2)

The comparison between pulses Pvp and Pfp provides the error pulse Pe that forces the VCO to synchronize the input. The block Pvp is an asynchronous circuit (Fig.9).

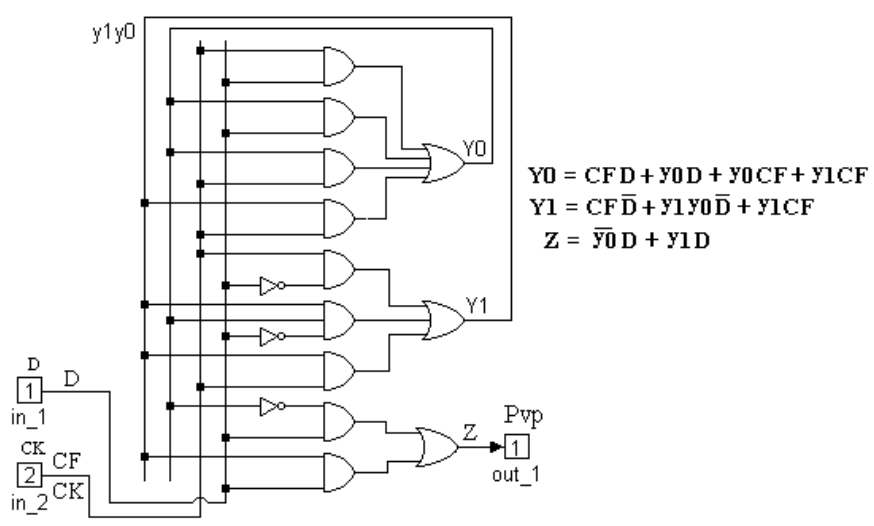

Fig.9 Intern aspect of the block Pvp

Fig.10 shows the waveforms of the proposed manual (equal to the corresponding synchronous version) [3]. 


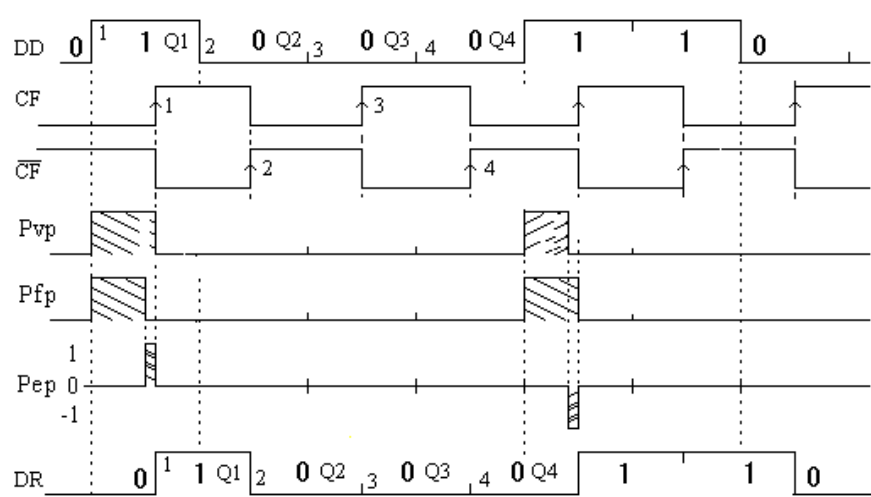

Fig.10 Waveforms of the asynchronous positive at half rate manual

The error pulse Pe diminishes during the synchronization time and disappear at the equilibrium point.

\section{B. Proposed by positive half rate automatic(ap-a/2)}

The block Pvp, common, produces the variable pulse Pvp between input and VCO. The block Pfp, shown below, produces the comparison fixed pulse Pfp (Fig.11).

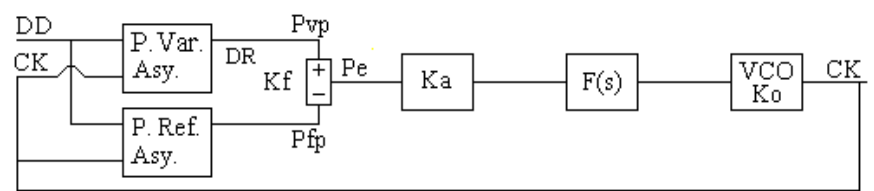

Fig.11 Asynchronous by both at half rate and automatic (ab-a/2)

The comparison between the pulses Pvp and Pfp provides the error pulse Pe that forces the VCO to follow the input. The block Pfp is an asynchronous circuit (Fig.12).

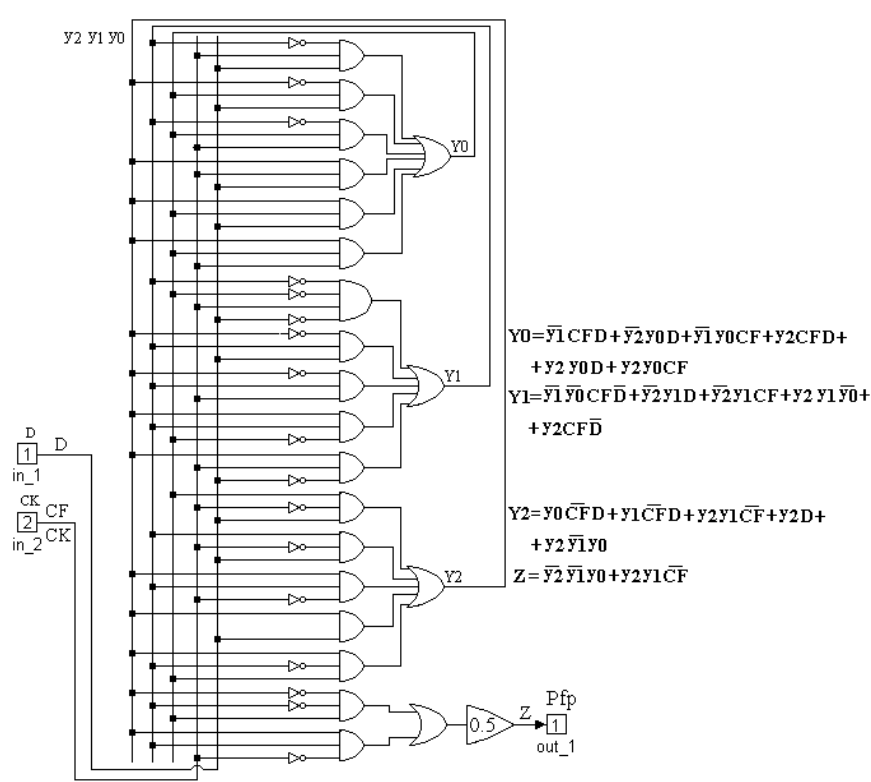

Fig.12 Intern aspect of the block Pfp

Fig.13 shows the waveforms of the proposed automatic (equal to the corresponding synchronous version) [3].

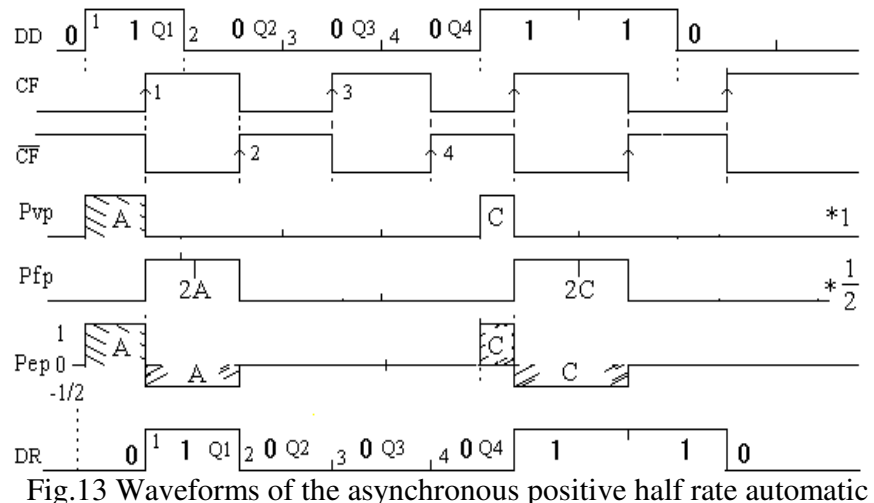

The error pulse Pe don't disappear, but the variable area Pv is equal to the fixed Pf at the equilibrium point.

\section{DESIGN, TESTS AND RESULTS}

We present the design, tests and results of the various synchronizers [5].

\section{A. Design}

To have guaranteed results, is necessary to dimension all the synchronizers with equal conditions. Then, the loop gain $\mathrm{Kl}=\mathrm{KdKo}=\mathrm{KaKfKo}$ must be equal in all the synchronizers. The phase detector gain Kf and the VCO gain Ko are fixed. Then, the loop gain amplification Ka controls the root locus and consequently the loop characteristics.

For analysis facilities, we use normalized values for the transmission rate $t x=1$ baud, clock frequency $\mathrm{fCK}=1 \mathrm{~Hz}$, extern noise bandwidth $\mathrm{Bn}=5 \mathrm{~Hz}$ and loop noise bandwidth $\mathrm{Bl}=0.02 \mathrm{~Hz}$. Then, we apply a signal power $\mathrm{Ps}=\mathrm{A}_{\text {ef }}^{2}$ and $\mathrm{a}$ noise power $\mathrm{Pn}=\mathrm{No}=2 \sigma \mathrm{n}^{2} . \Delta \tau$, where $\sigma \mathrm{n}$ is the noise standard deviation and $\Delta \tau=1 /$ fSamp is the sampling period. The relation between SNR and noise variance $\sigma \mathrm{n}^{2}$ is

$\mathrm{SNR}=\mathrm{A}_{\text {ef }}^{2} /(\mathrm{No} . \mathrm{Bn})=0.5^{2} /\left(2 \sigma \mathrm{n}^{2} * 10^{-3} * 5\right)=25 / \sigma \mathrm{n}^{2}$

Now, for each synchronizer, is necessary to measure the output jitter UIRMS versus the input SNR

- $1^{\text {st }}$ order loop:

We use a cutoff loop filter $\mathrm{F}(\mathrm{s})=0.5 \mathrm{~Hz}$, is 25 times greater than $\mathrm{Bl}=0.02 \mathrm{~Hz}$, what eliminates the high frequency but maintain the loop characteristics. The transfer function is

$$
H(s)=\frac{\mathrm{G}(\mathrm{s})}{1+\mathrm{G}(\mathrm{s})}=\frac{K d K o F(s)}{s+K d K o F(s)}=\frac{K d K o}{s+K d K o}
$$

the loop noise bandwidth is

$$
\mathrm{Bl}=\frac{K d K o}{4}=K a \frac{K f K o}{4}=0.02 \mathrm{~Hz}
$$

So, with $(\mathrm{Km}=1, \mathrm{~A}=1 / 2, \mathrm{~B}=1 / 2, \mathrm{Ko}=2 \pi)$ and loop bandwidth $\mathrm{Bl}=0.02$, we obtain respectively the $\mathrm{Ka}$, for analog, hybrid, combinational and sequential synchronizers, then

$$
\begin{aligned}
& B l=(K a . K f . K o) / 4=(K a \cdot K m \cdot A \cdot B \cdot K o) / 4->K a=0.08 * 2 / \pi \\
& B l=(K a \cdot K f . K o) / 4=(K a \cdot K m \cdot A \cdot B \cdot K o) / 4->K a=0.08 * 2.2 / \pi \\
& B l=(K a \cdot K f . K o) / 4=\left(K a * 1 / \pi^{*} 2 \pi\right) / 4->K a=0.04 \\
& B l=(K a . K f . K o) / 4=\left(K a * 1 / 2 \pi^{*} 2 \pi\right) / 4->K a=0.08
\end{aligned}
$$

For the analog PLL, the jitter is

$$
\sigma_{\phi}^{2}=\text { Bl.No/Aef } f^{2}=0.02 * 10^{-3} * 2 \sigma \mathrm{n}^{2} / 0.5^{2}=16^{*} 10^{-5} . \sigma \mathrm{n}^{2}
$$

For the others PLLs, the jitter formula is more complicated. $-2^{\text {nd }}$ order loop:

Is not used here, but provides similar results. 


\section{B. Tests}

We used the following setup to test synchronizers (Fig.14)

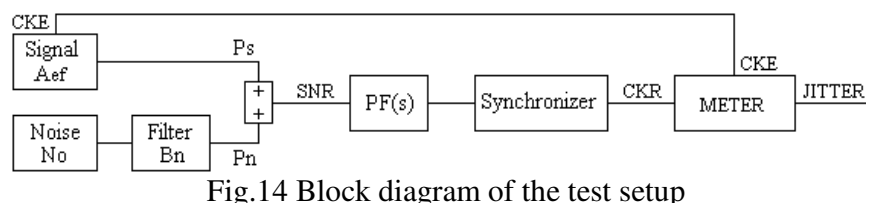

The receiver recovered clock with jitter is compared with the emitter original clock, the difference is the jitter.

\section{Results}

We present the results in terms of output jitter UIRMS versus input SNR. Fig. 15 shows the jitter - SNR curves of the four synchronizers which are the both rate manual (ab-m), the both rate automatic (ab-a), the positive half rate manual (ap$\mathrm{m} / 2$ ) and the positive half rate automatic (ap-a/2).

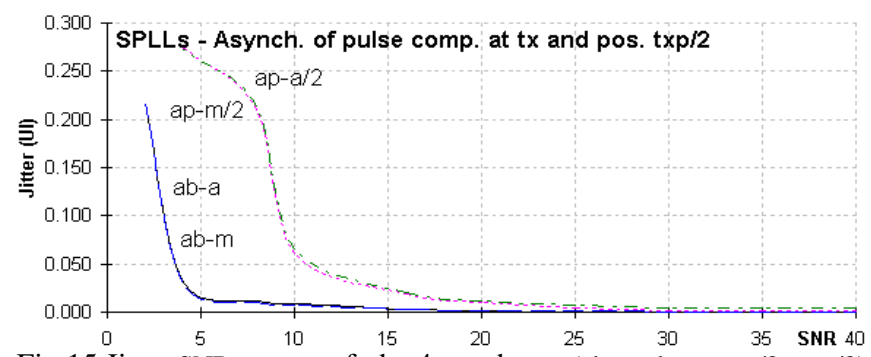

Fig. 15 Jitter-SNR curves of the 4 synchron. (ab-m,ab-a,ap-m/2,ap-a/2)

We observe that, in general, the output jitter UIRMS decreases gradually with the input SNR increasing.

We verify that, for high SNR, the four jitter curves tend to be similar. However, for low SNR, the variant asynchronous both at rate manual (ab-m) and automatic (ab-a) are better than the variant asynchronous positive at half rate manual (ap-m/2) and automatic (ap-a/2).

\section{CONCLUSIONS}

We studied four synchronizers involving the reference variant asynchronous by both transitions at rate with versions manual (ab-m) and automatic (ab-a) and the proposed variant asynchronous by positive transitions at half rate with versions manual (ap-m/2) and automatic (ap-a/2). Then, we tested and compared their jitter - SNR curves.

We observed that, in general, the output UIRMS jitter curves decrease gradually with the input SNR increasing.

We verified that, for high SNR, the four synchronizers jitter curves tend to be similar, this is comprehensible since all the synchronizers are digital, with equal noise margin. However, for low SNR, the variant asynchronous by both at rate with their versions manual (ab-m) and automatic (ab-a) are better than the variant asynchronous by positive at half rate with their versions manual (ap-m/2) and automatic (ap-a/2), this is comprehensible because the variant by both transitions at rate has less states than the variant by positive transitions at half rate and then, in the 1st case, the time to pass from the error state to the correct state is lesser.

In the future, we are planning to extend the present study to other types of synchronizers.

\section{ACKNOWLEDGMENTS}

The authors are grateful to the program FCT (Foundation for sCience and Technology) / POCI2010.

\section{REFERENCES}

[1] J. C. Imbeaux, "performance of the delay-line multiplier circuit for clock and carrier synchronization", IEEE Jou. on Selected Areas in Communications p.82 Jan. 1983.

[2] Werner Rosenkranz, "Phase Locked Loops with limiter phase detectors in the presence of noise", IEEE Trans. on Communications com-30 Nº 10 pp.2297-2304. Oct 1982.

[3] H. H. Witte, "A Simple Clock Extraction Circuit Using a Self Sustaining Monostable Multivibrat. Output Signal", Electronics Letters, Vol.19, Is.21, pp.897-898, Oct 1983.

[4] Charles R. Hogge, "A Self Correcting Clock Recovery Circuit", IEEE Tran. Electron Devices p.2704 Dec 1985.

[5] A. D. Reis, J. F. Rocha, A. S. Gameiro, J. P. Carvalho "A New Technique to Measure the Jitter", Proc. III Conf. on Telecommunications pp.64-67 FFoz-PT 23-24 Apr 2001.

[6] Marvin K. Simon, William C. Lindsey, "Tracking Performance of Symbol Synchronizers for Manchester Coded Data", IEEE Transactions on Communications Vol. com-2.5 No4, pp.393408, April 1977.

[7] J. Carruthers, D. Falconer, H. Sandler, L. Strawczynski, "Bit Synchronization in the Presence of Co-Channel Interference", Proc. Conf. on Electrical and Computer Engineering pp.4.1.14.1.7, Ottawa-CA 3-6 Sep. 1990.

[8] Johannes Huber, W. Liu "Data-Aided Synchronization of Coherent CPM-Receivers" IEEE Transactions on Communications Vol.40 Nº1, pp.178-189, Jan. 1992.

[9] Antonio D'Amico, A. D'Andrea, Reggianni, "Efficient NonData-Aided Carrier and Clock Recovery for Satellite DVB at Very Low SNR", IEEE Jou. on Sattelite Areas in Comm. Vol.19 No12 pp.2320-2330, Dec. 2001.

[10] Rostislav Dobkin, Ran Ginosar, Christos P. Sotiriou "Data Synchronization Issues in GALS SoCs", Proc. 10th International Symposium on Asynchronous Circuits and Systems, pp.CD-Ed., Crete-Greece 19-23 Apr. 2004.

[11] N. Noels, H. Steendam, M. Moeneclaey, "Effectiveness Study of Code-Aided and Non-Code-Aided ML-Based Feedback Phase Synchronizers", Proc. IEEE Int Conf. on Comm.(ICC'06) pp.2946-2951, Ist.-TK, 11-15 Jun 2006.

[12] A. D. Reis, J. F. Rocha, A. S. Gameiro, J. P. Carvalho "Sequential Symbol Synchronizers based on Clock Sampling of Discrete Pulses", Proc. VIII Symposium on Enabling Optical Network and Sensors (SEONs) p.CD-Edited, Porto-PT 25-25 June 2010.

[13] A. D. Reis, J. F. Rocha, A. S. Gameiro, J. P. Carvalho "Optical Digital Communication Systems and Synchronism", Proc. 7th UBI International Conference on Engeneering (for Economic Development) - ICEUBI 2013, pp. CT8-11.7, Covilhã-PT 27-29 November 2013. 